\title{
Das Elbe-Urstromtal im Bereich der Deutschen Bucht (Nordsee)
}

\author{
Klaus Figge *)
}

\author{
Channel (Urstromtal), Upper Pleistocene, marin sediment, marin relief \\ Holocene (base), section. North Sea (German Bight)
}

Kurzfassung: Mit Hilfe sedimentechographischer Messungen wurde in der Deutschen Bucht nordwestlich von Helgoland unter dem Holozän eine breite Rinne nachgewiesen, die die nördliche Fortsetzung von Elbe- und Weser-Urstromtal ist. Die Rinnensohle fällt im Untersuchungsgebiet nach $\mathrm{NW}$ von SKN $-38 \mathrm{~m}$ auf SKN $-56 \mathrm{~m}$ ab, die Holozänbedeckung nimmt in gleicher Richtung von 4,5 auf $16 \mathrm{~m}$ zu. Das Tal wird von einer $3,5 \mathrm{~m}$ bis $10 \mathrm{~m}$ höher gelegenen Terrasse begleitet.

\section{[The Pleistocene Watercourse of the Elbe in the Region of the German Bight (North Sea)]}

A bstract: In the German Bight northwest of Heligoland a wide trough has been located below Holocene deposits, using sub-bottom-profiling techniques. It is the northern extension of the Elbe- and Weser-urstromtal. In the surveyed area, the valley floor dips towards NW from $38 \mathrm{~m}$ to $56 \mathrm{~m}$ below SKN. The Holocene cover increases in the same direction from $4.5 \mathrm{~m}$ to $16 \mathrm{~m}$. The trough is paralleled by a terrace at an elevation between $3.5 \mathrm{~m}$ and $10 \mathrm{~m}$. The thickness of the Holocene cover increases in the same direction from 4.5 to $16 \mathrm{~m}$.

\section{Einleitung}

Im Rahmen der Erledigung dienstlicher Aufgaben ergab sich beim Deutschen Hydrographischen Institut die Notwendigkeit, die Mächtigkeit der holozänen Sedimente in der Deutschen Bucht zu untersuchen. Aus diesem Grund wurden zwischen 1975 und 1979 ca. 1200 Seemeilen Profile mit einem Sedimentechographen abgefahren, mit denen die Basis des Holozäns im Gesamtgebiet zwar noch nicht genügend genau erfaßt werden konnte, die aber für Teilbereiche wie das Elbe-Urstromtal, das sich von Helgoland aus nach NW erstreckt, bereits jetzt einen guten Überblick über den Aufbau der obersten Sedimentschichten geben (Abb. 1).

\section{Methodisches}

Für die Messungen kam ein Sedimentechograph vom Typ „UNIBOOM“ der Firma EG \& G zum Einsatz, die Registrierungen erfolgten mit einem EPC-Recorder Modell 4100. Die Anordnung der Geräte ist in Abbildung 2 skizziert: Von einem Meßschiff (VFS „GAUSS“) wurde bei langsamer Fahrt ein auf einem Katamaran montierter Schwinger knapp unter der Meeresoberfläche geschleppt, der Schallimpulse mit einer Energie von $300 \mathrm{~J}$ im Abstand von einer halben Sekunde bzw. von $200 \mathrm{~J}$ bei $1 / 4$ Sekunde abgab. Die Frequenz des Impulses liegt nach Herstellerangaben zwischen $400 \mathrm{~Hz}$ und $14 \mathrm{kHz}$, das Energiemaximum dürfte bei $1,2 \mathrm{kHz}$ liegen. Als Empfänger dienten 8 in einer 1,5 $\mathrm{m}$ langen Kette hintereinander angeordnete Hydrophone, die durch ein Kabel mit der Registriereinheit an Bord verbunden waren.

*) Anschrift des Verfassers: Dr. K. Figg e, Deutsches Hydrographisches Institut, BernhardNocht-Straße 78, 2000 Hamburg 4. 


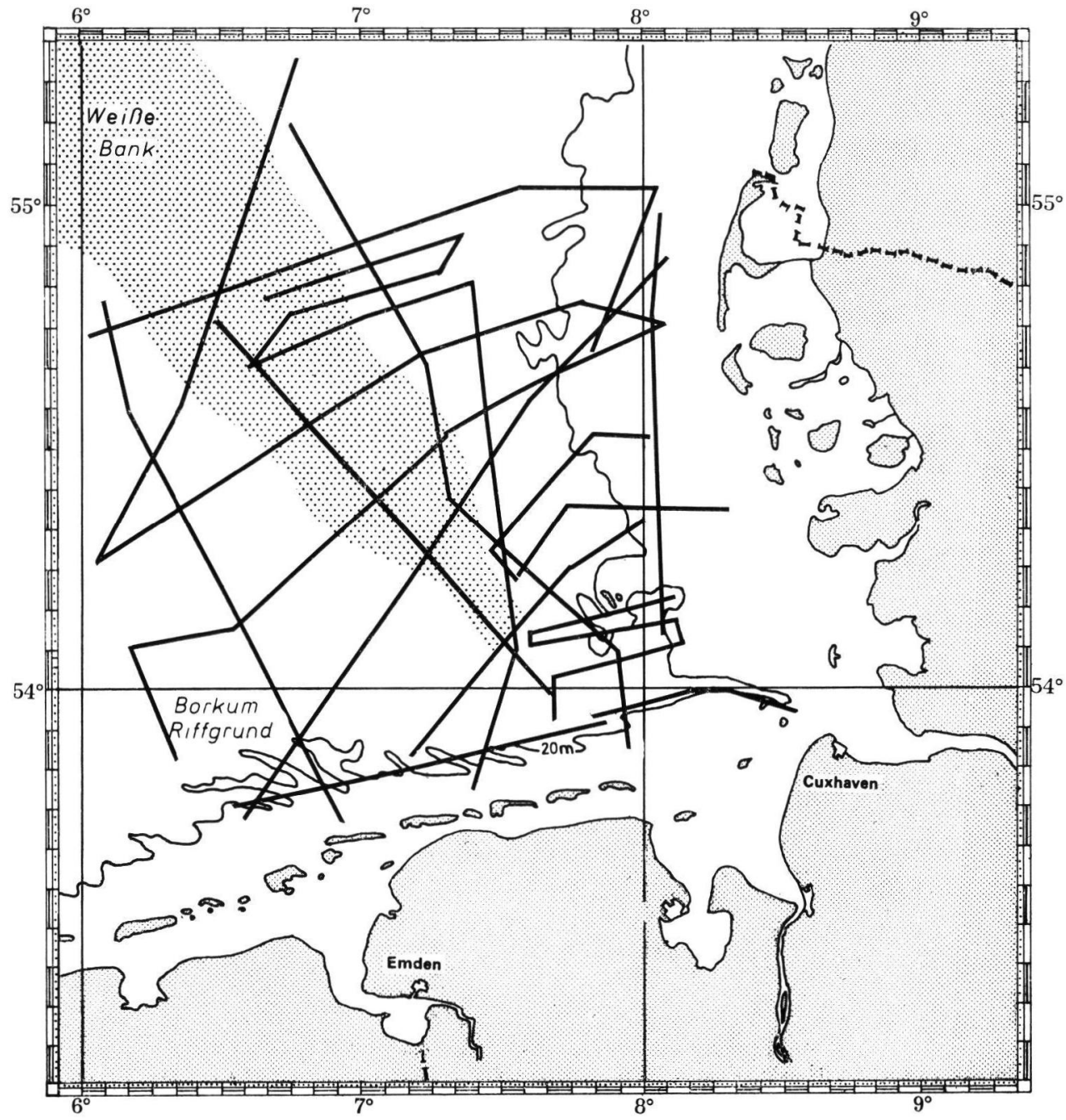

Abb. 1: Arbeitsgebiet und Lage der Profile.

Leider sind die Nutzsignale sehr stark durch Schiffsgeräusche und Seegang gestört. Eine Verbesserung der Aufzeichnungen konnte zwar durch Zwischenschalten eines BandPass-Filters mit den Grenzen $400 \mathrm{~Hz}$ und $3,6 \mathrm{kHz}$ erreicht werden, bei Windstärken oberhalb 5 Beaufort lag der Störpegel jedoch so hoch, daß die Messungen abgebrochen werden mußten.

Bei den Auswertungen wurde für die holozänen Lockersedimente in Anlehnung an Schirmer, Schmalfeld \& Siebert (1979) eine Schallgeschwindigkeit von $1600 \mathrm{~m} / \mathrm{s} \mathrm{zu}$ grunde gelegt, in den abgebildeten Sedimentechogrammen entsprechen $10 \mathrm{~ms}$ mithin einer Schichtdicke von $8 \mathrm{~m}$ (doppelte Wegstrecke). Der Meßfehler liegt bei $\pm 30 \mathrm{~cm}$. Die Positionsbestimmungen erfolgten nach Decca. Die Angaben über die heutigen Wassertiefen wurden der Seekarte D 50 entnommen. 


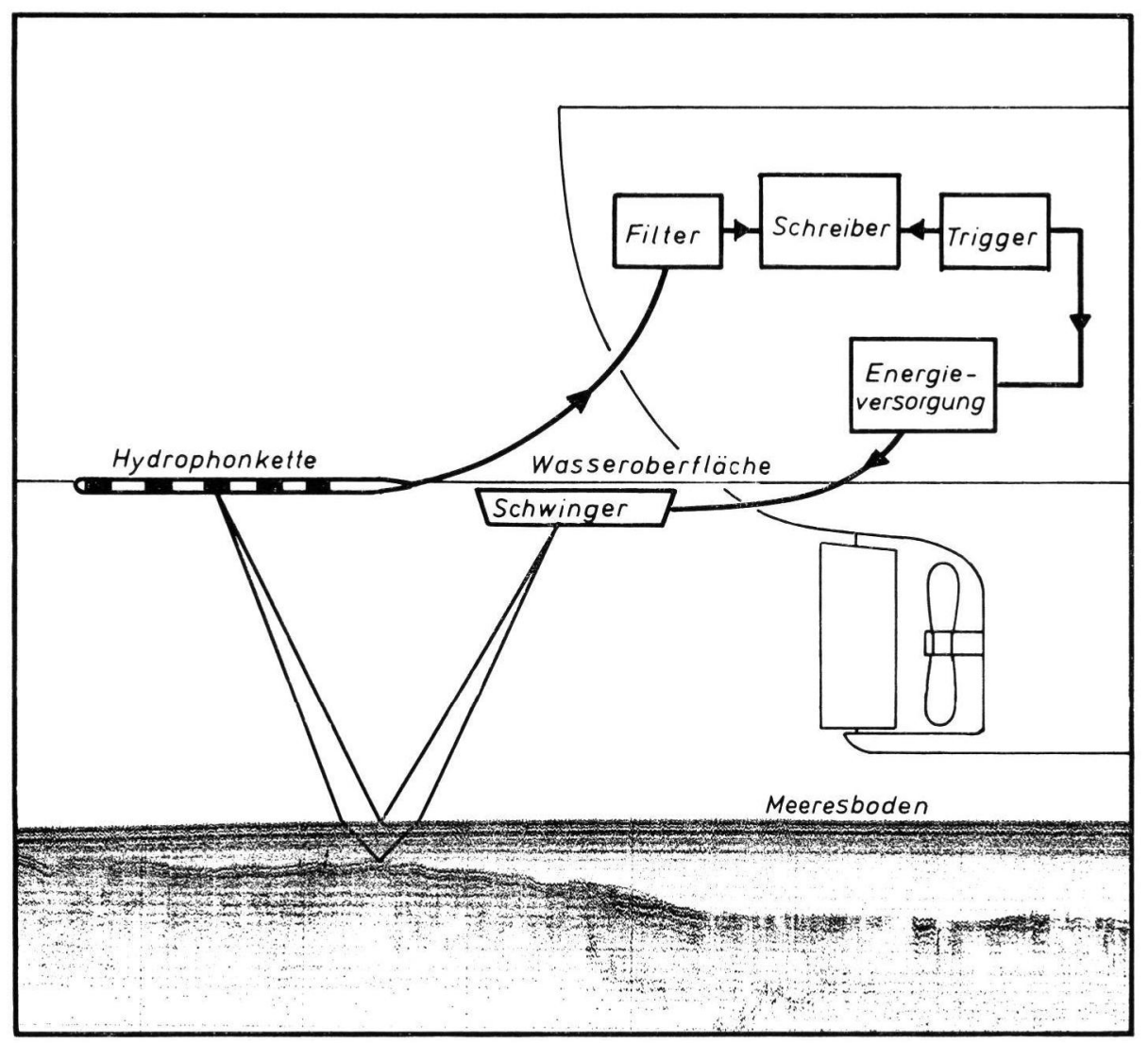

Abb. 2: Schematische Darstellung des Meßsystems.

\section{Registrierungen}

Im Rahmen des hier behandelten Themas sind lediglich zwei Reflektorhorizonte von Bedeutung:

1. Horizont „M“ (Meeresboden):

Dieser Horizont spiegelt den großräumigen morphologischen Aufbau der Deutschen Bucht außerhalb des Küstenbereichs wider, in dem drei Strukturelemente hervortreten:

Der Borkum-Riffgrund, eine Hochlage pleistozäner Gesteine, bei dem die Einebnung durch Seegang und Strömungen der heutigen Nordsee noch nicht sehr weit fortgeschritten ist und der noch ein lebhaftes Relief aufweist. Eine engmaschige Kartierung der Sedimentoberfläche (Karte des Seegrundes, $1: 100$ 000) zeigt einen raschen Wechsel von Feinsand, Grobsand und Kies, wobei die gröberen Fraktionen vorherrschen.

Das rechte Ufer des Elbe-Urstromtales, das von Helgoland aus nach NW verläuft und den Tiefenbereich etwa zwischen SKN $-40 \mathrm{~m}$ bis SKN $-25 \mathrm{~m}$ einnimmt (SKN = mittleres Springniedrigwasser, am Pegel Helgoland z. B. 1,75 m unter NN). Wie beim Borkum-Riffgrund ist das Relief lebhaft, wobei uferparallele Strukturen vorherrschen, und wie dort wechseln die Sedimenttypen auf engstem Raum. 

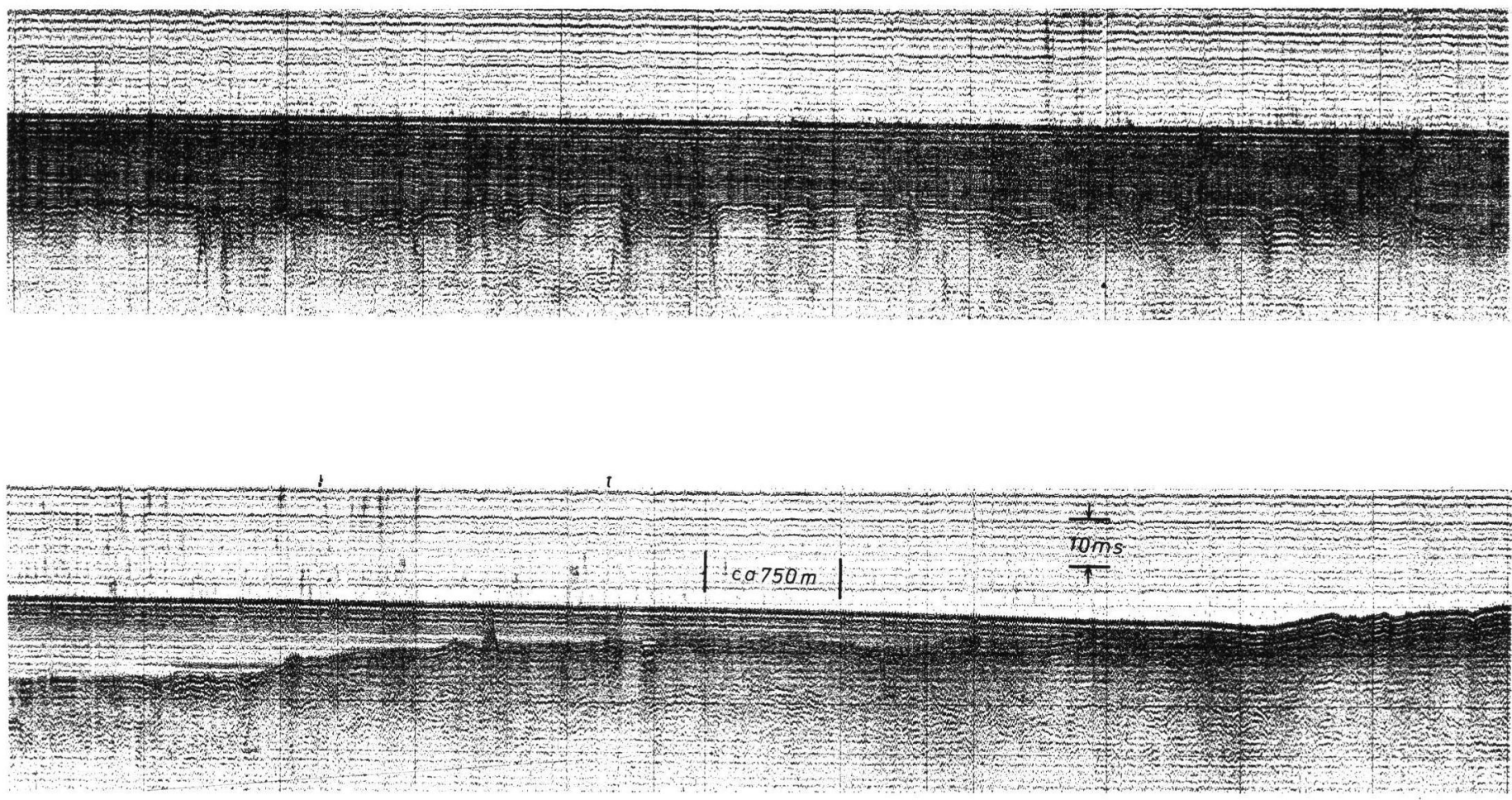

Abb. 3: Querprofile durch das Elbe-Urstromtal. Oben: Flußmitte (Profil 7). Unten: Ostufer mit Terrasse (Profil 7). 

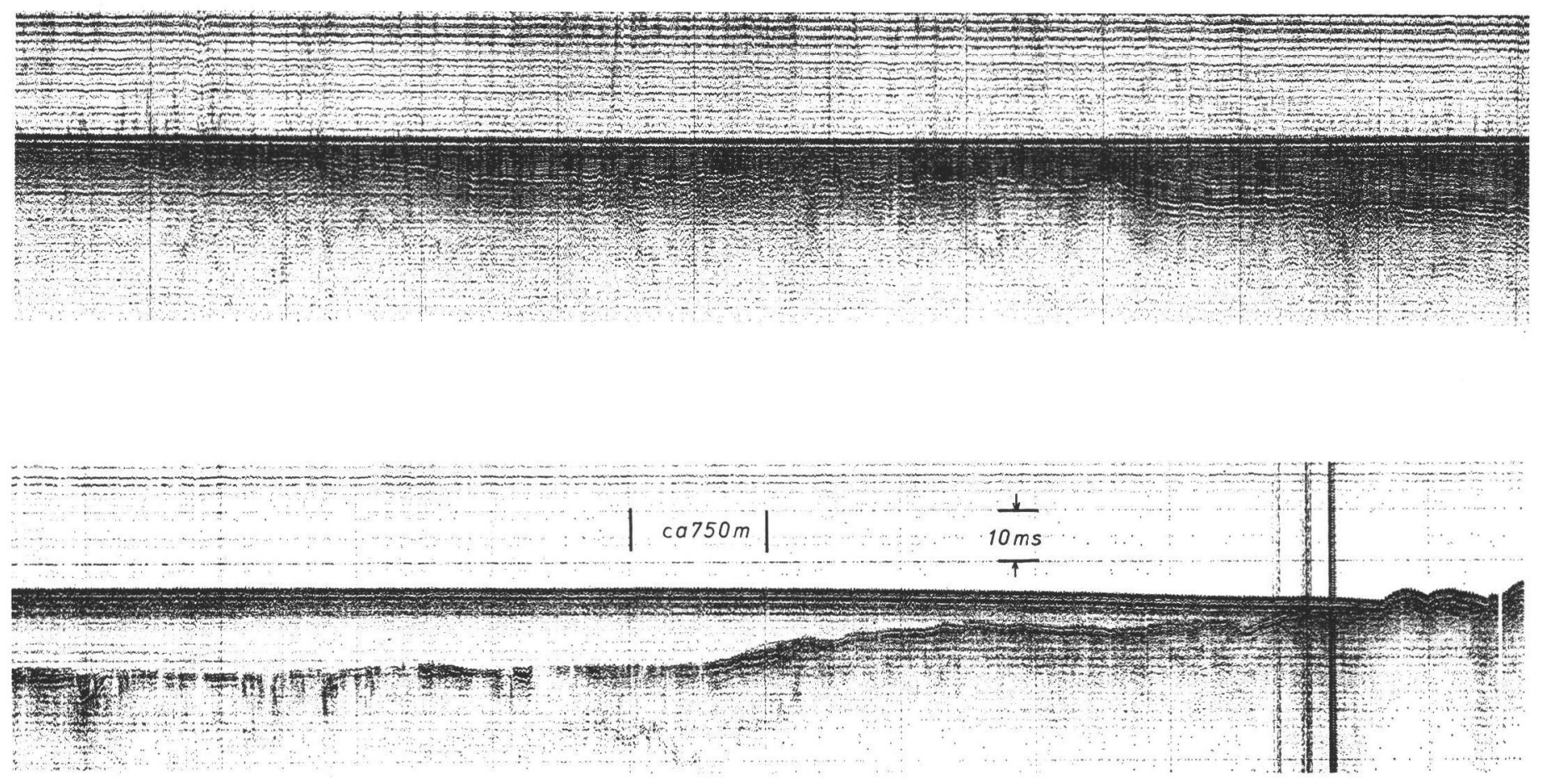

Abb. 4: Terrasse. Oben: Westufer (Profil 7). Unten: Ostufer (Profil 19) mit Übergang zur ehemaligen Geest (Erhebungen ganz rechts). Die dicken senkrechten Linien sind durch Funkverkehr entstandene Störungen. 
Der sich westlich an das Elbufer und nördlich an den Borkum-Riffgrund anschließende Meeresboden bietet in den Echogrammen ein über weite Strecken ruhiges Bild. Seine Tiefenlage nimmt nach NW langsam zu. Die Sedimente bestehen aus Feinsand und siltigem Feinsand.

\section{Horizont „ $\mathrm{H}_{\mathrm{o}}$ “ (Basis des Holozäns)}

Dieser Reflektor ist fast ebenso markant wie die Meeresbodenoberfläche und weist damit auf einen signifikanten Dichteunterschied zwischen dem Holozän und dem Liegenden hin. Die Alterseinstufung als Holozänbasis konnte im Bereich des Elbtals bis jetzt noch nicht durch Bohrungen bewiesen werden, erscheint aber kaum zweifelhaft, da sich der Reflektor in allen Profilen bis zum östlichen Elbufer bzw. zum Borkum-Riffgrund verfolgen läßt, wo er entweder am Meeresboden ansteht oder in Bohrungen unter einer dünnen Decke holozäner Sedimente angetroffen wurde (Bäsemann 1979; Ludwig, Müller \& STREIF 1979).

Das großräumige Bild dieses Horizontes ist im Bereich des Elbtales eine bemerkenswert ebene, nach NW geneigte Fläche, die sich zur Flußmitte leicht absenkt. Nur gelegentlich finden sich sanfte Erhebungen und Senken mit schwach geneigten Flanken (Abb. 3). Der flache Anstieg zum früheren Ufer wird auf beiden Seiten durch eine unterschiedlich breite, selten aber fehlende Terrasse unterbrochen (Abb. 4).

Im Detail läßt der Reflektor besonders im Bereich des Flußbettes zahlreiche Unregelmäßigkeiten erkennen. In den Registrierungen wird der Reflex an einigen Stellen abrupt sehr schwach (Abb. 3), an anderen Stellen wiederum finden sich besonders intensive Schwärzungen. Diese Effekte sind nicht apparativ bedingt, sondern müssen ihre Ursache in lokal begrenzten Materialunterschieden haben. Recht häufig findet man auch ehemalige "Löcher", wahrscheinlich Kolke, die offensichtlich durch den Fluß selber zugeschüttet wurden und z. T. mehrere Reflexionen übereinander zeigen, deren oberste im Niveau der Flußsohle liegt. Der Abstand der Profile voneinander ist so groß, daß aus den in den Querprofilen angetroffenen "Löchern" nicht auf das Vorhandensein von Rinnen geschlossen werden kann.

\section{Ergebnis}

Bereits früher wurde von verschiedenen Autoren aufgrund geologischer und morphologischer Betrachtungen im Bereich der Deutschen Bucht die Existenz eines Vorfluters angenommen, in den Elbe, Weser und die von Schleswig-Holstein nach W hin entwässernden Schmelzwasserrinnen einmündeten. Nach Valentin (1957) handelt es sich um einen Eisstausee, der sich von Helgoland trichterförmig nach WNW erweiterte. REINHARD (1974) legt die Mündung des Elbe-Urstroms in den Raum des Austerngrundes. Die vorliegenden Meßergebnisse erlauben es nun, Lage und Verlauf des Elbe-Urstroms zwischen Helgoland und der Weißen Bank (etwa $55^{\circ} \mathrm{N}$ )zu präzisieren.

In Abbildung 5 ist die Tiefenlage der Holozänbasis wiedergegeben, die das Flußbett als eine sich von ca. $25 \mathrm{~km}$ auf ca. $40 \mathrm{~km}$ nach NW verbreiternde Rinne erkennen läßt. Das rechte Ufer ist steil und leitet zur ehemaligen Geest über, an das linke schließen sich weite Flächen an, die auch in Sedimentechogrammen außerhalb des Arbeitsgebietes gefunden wurden und dem morphologischen Bild der heutigen Nordsee entsprechen. Ca. $52 \mathrm{~km}$ nordwestlich Helgoland mündete ein Nebenfluß in die Elbe, dessen Bett sich bis in das Gebiet nördlich des Helgoländer Steingrundes verfolgen läßt und der möglicherweise mit der heutigen Eider in Verbindung gebracht werden kann.

Die Tiefe der Sohle liegt in Flußmitte etwa $2 \mathrm{~m}$ tiefer als an den Rändern und beträgt südwestlich Helgoland SKN $-38 \mathrm{~m}$, bei der Weißen Bank SKN $-56 \mathrm{~m}$. Daraus ergibt sich ein Gefälle von $18 \mathrm{~m}$ auf einer Strecke von $106 \mathrm{~km}$ oder der hohe Wert von 0,17 0\% 


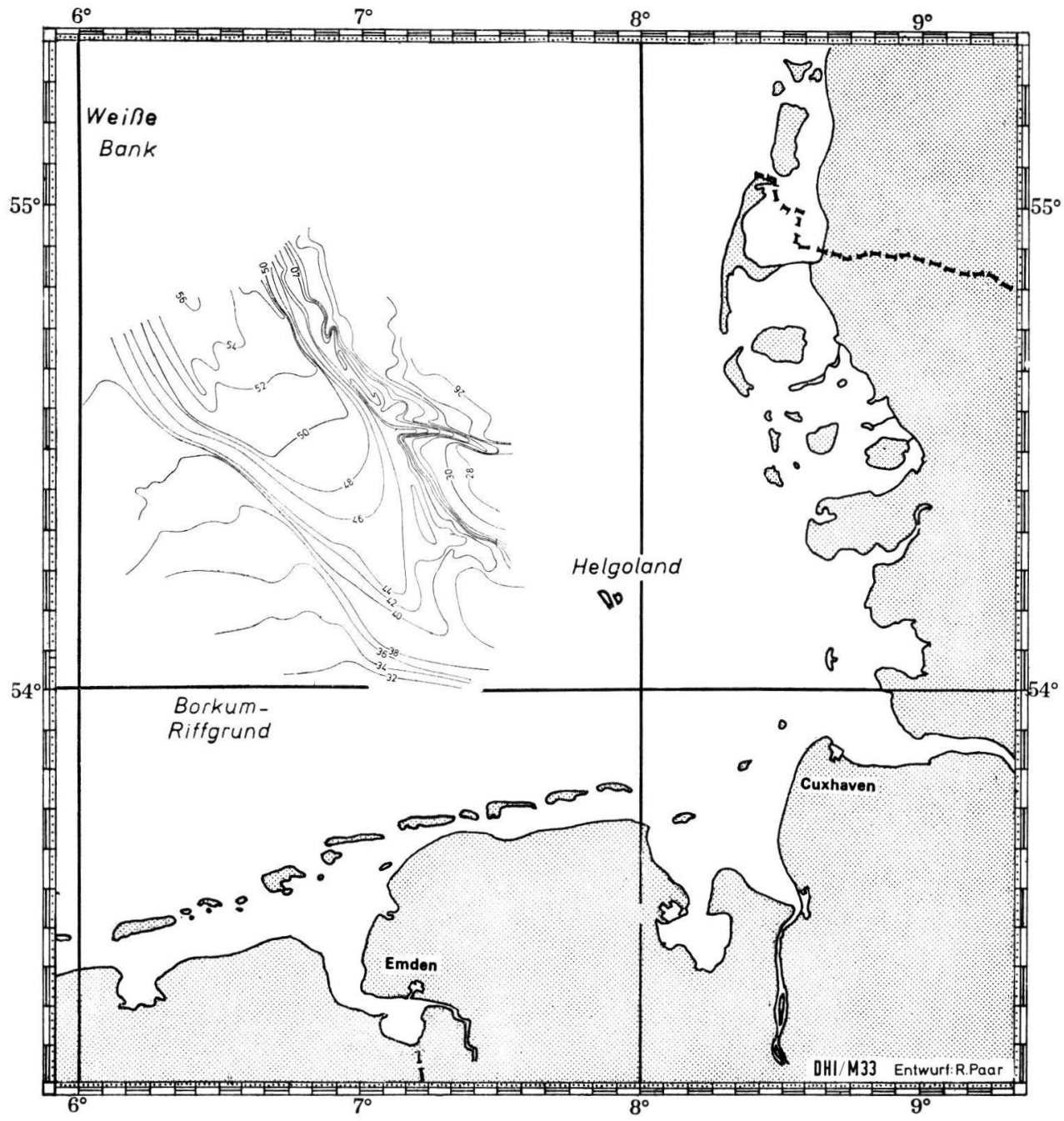

Abb. 5: Tiefenlage der Holozänbasis im Elbe-Urstromtal in Metern unter SKN. Entfernung $54^{\circ}$ bis $55^{\circ}: 60 \mathrm{sm} \approx 111 \mathrm{~km}$.

Ein noch steileres Gefälle errechnet sich für den Bereich zwischen dem SKN $-38 \mathrm{~m} \mathrm{Ni}$ veau und der Tiefe der heutigen Elbe, die nördlich von Scharhörn bei einer Tiefe von ungefähr SKN -20 m präholozäne Schichten anschneidet. Ohne Berücksichtigung einer späteren Erosion kommt man allein aus diesen Zahlen auf einen Wert von $18 \mathrm{~m}$ auf $68 \mathrm{~km}$ oder $0,27 \%$ oo. Es muß einer späteren Untersuchung vorbehalten bleiben, diese hohen Beträge zu deuten.

Das Ufer des Urstromtals wird beidseitig von einer unterschiedlich breiten Terrasse begleitet, die westlich Helgoland ca. 3,5 m und in Höhe der Weißen Bank fast $10 \mathrm{~m}$ über der Flußsohle (Mitte) liegt. Sie taucht damit ebenfalls nach NW ab, aber in geringerem Winkel als das Flußbett. Stellenweise scheint sich in einigen Echogrammen eine zweite 
Terrasse über der erstgenannten anzudeuten, jedoch sind die vorliegenden Informationen für eine zuverlässige Aussage darüber noch nicht ausreichend.

Die oben erwähnten schwachen Erhebungen und Senken im Flußbett überschreiten selten $2 \mathrm{~m}$ und werden als regional begrenzte Sandbänke oder lokale Eintiefungen gedeutet.

Es entsteht die Frage nach der südlichen Fortsetzung der Rinne und ihre Anknüpfung an das Gewässernetz des heutigen Festlandes. Leider ist hierfür ein Tiefenbereich anzunehmen, in dem durch die nacheiszeitliche Nordsee eine flächenhafte Abrasion stattgefunden hat, deren Folge eine weitgehende Einebnung des früheren Reliefs ist. Keines der z. Zt. aus dem Gebiet südlich Helgoland zur Verfügung stehenden Sedimentechogramme läßt dann auch eine alte Rinne mit Sicherheit erkennen. Es wurden auch keine Hinweise auf einen Zusammenfluß von Weser und Elbe gefunden, obwohl zu vermuten ist, daß

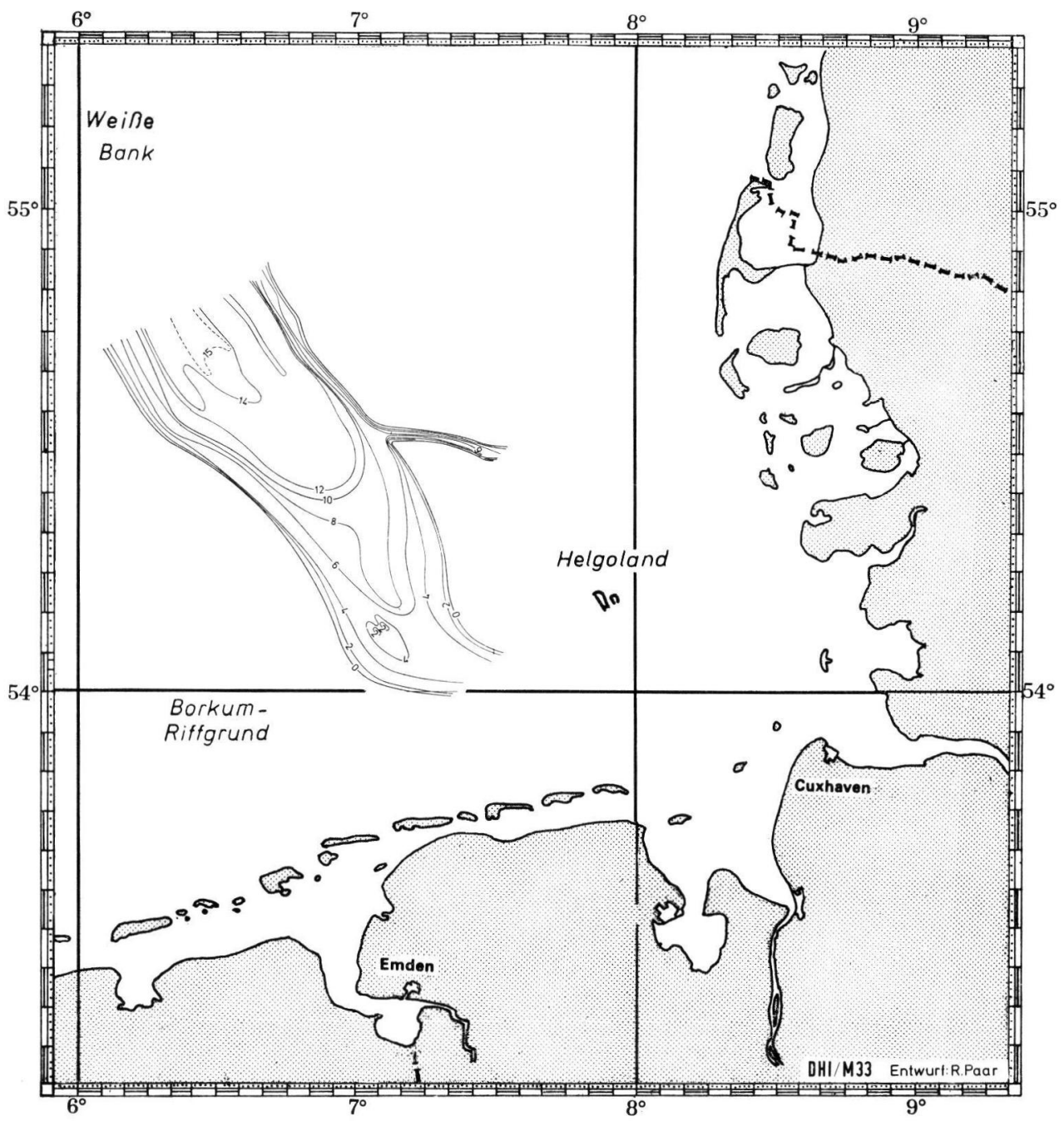

Abb. 6: Mächtigkeit des Holozäns im Elbe-Urstromtal. Außerhalb der 0 m-Linie dünner, nicht meßbarer Schleier mariner Sande möglich, nach NW an Mächtigkeit zunehmend. 
die Weser südwestlich von Helgoland in das Elbe-Urstromtal mündete, denn es erscheint unwahrscheinlich, daß dieser Fluß nach $W$ abgeknickt und über den Borkum-Riffgrund geflossen ist.

Die Mächtigkeit des Holozäns ist in der Abbildung 6 dargestellt. Sie beträgt südwestlich Helgoland 4,5 m und wächst in Richtung auf die Weiße Bank auf $16 \mathrm{~m}$ an. Aus den Sedimentechogrammen kann nicht auf die Art der Sedimente geschlossen werden, da jedoch deutliche Impedanzsprünge fehlen, ist zumindest mit einer petrographischen Gleichförmigkeit des gesamten Schichtpaketes zu rechnen. Nach Ludwig, MüLleR \& Streif (1979) sind in dieser Serie mehrere stratigraphische Horizonte zu vermuten.

Leider war es wegen ungünstiger Wetterbedingungen nicht möglich, die Untersuchungen weiter nach NW auszudehnen. Ich hoffe, daß hierzu in den nächsten Jahren Gelegenheit besteht.

\section{Schriftenverzeichnis}

BäSEMANN, H. (1979): Feinkiesanalytische und morphologische Untersuchungen an Oberflächensedimenten der Deutschen Bucht. - Diss. Univ. Hamburg, 143 S., 27 Abb., 12 Kt.; Hamburg.

JARKE, J. (1957): Neue Ergebnisse zur Bodenbedeckung der Deutschen Bucht. - Abh. 30. Geographentag Hamburg 1955, 369-375, 1 Abb., 1 Kt.; Hamburg.

Ludwig, G., Müller, H. \& Streif, H. (1979): Neuere Daten zum holozänen Meeresspiegelanstieg im Bereich der Deutschen Bucht. - Geol. Jb., D 32: 3-22, 2 Abb., 7 Tab.; Hannover.

Pratje, O. (1951): Die Deutung der Steingründe in der Nordsee als Endmoränen. - Dt. Hydrogr. Z., 4: 201-205; Hamburg.

Reinhard, H. (1974): Genese des Nordseeraumes im Quartär. - Fennia, 129, 95 S., 10 Abb., 2 Beil.; Helsinki.

Schirmer, F., Schmalfeld, B. \& Siebert, J. (1979): Schallgeschwindigkeit und Impedanz des oberen Meeresbodens in Gebieten der Nordsee, des Skagerraks und der Ostsee. - Dt. Hydrogr. Z., 32: 279-288, 5 Abb.; Hamburg.

Sindowski, K.-H. (1970): Das Quartär im Untergrund der Deutschen Bucht (Nordsee). - Eiszeitalter u. Gegenwart, 21: 33-46, 8 Abb., 8 Tab.; Ohringen.

Valentin, H. (1957): Die Grenze der letzten Vereisung im Nordseeraum. - Abh. 30. Geographentag Hamburg 1955, 359-366, 4 Kt.; Hamburg.

Karte des Seegrundes $1: 100000$, Deutschland, Nordsee, Serie M 644, Blatt 1-8, Hrg. Militärgeographisches Amt, Bonn-Bad Godesberg.

Manuskript eingegangen am 23. 1. 1980. 
